\title{
THE EFFECTS OF ETHANOL INTOXICATION ON PHOBIC ANXIETY*
}

\author{
Bruce A. Thyer $\dagger$ and George C. Curtis \\ Department of Psychiatry, The University of Michigan Hospitals, Ann Arbor, MI 48109, U.S.A.
}

(Received 25 January 1984)

\begin{abstract}
Summary-Behavioral conceptions of alcohol abuse often include the hypothesis that drinking behavior is a negatively reinforced operant, with ethanol intoxication viewed as alleviating aversive environmental and internal states. This hypothesis has not heen confirmed or refuted hy previous studies which employed mild stressors and limited assessment methodology. In the present experiment, 22 patients with severe phobias approached their phobic animal under two consecutive conditions-first while sober and second after drinking either a placebo or an intoxicating dose of ethanol. The severe anxiety induced was assessed behaviorally, physiologically and by the patient's self-report of fear. The intoxicated patients did not experience decreased anxiety, tachycardia or avoidance, compared to the placebo group. These results have clinical implications and suggest the need to reconsider tension-reduction theories of alcohol abuse.
\end{abstract}

\section{INTRODUCTION}

Of the available behavioral explanations for alcohol abuse, the tension-reduction theory (TRT) has been the subject of the majority of empirical investigations. The TRT states that ethanol consumption is a negatively reinforced operant response, with aversive affective or physiological states in the drinker being temporarily eliminated by the anxiolytic properties of the drug (Conger, 1956; Shoben, 1956; Kingham, 1958). The relative immediacy of the rewarding consequences of ethanol intoxication exerts a more potent influence on the individual's drinking behavior than the delayed aversive consequences of hangover or financial deprivation, thus perpetuating the cycle of alcohol abuse.

Despite dozens of experiments on the TRT, recent reviews do not agree about its validity. The conclusion arrived at by Cappell and Herman (1972) over a decade ago has not been substantially altered: ". . much of the evidence is negative, equivocal, and often contradictory" (p. 33). Brown and Crowell (1974) review studies of animal conflict-test experiments and state that "... it would be premature to conclude either that the conflict-test data confirm a tension-reduction conception or that the data from other tests render it untenable" (p. 83). On the other hand, Hodgson, Stockwell and Rankin (1979) have argued that, when an appropriate experimental paradigm has been employed. animal research supports the TRT. Reviews of the experimental human literature on the role personality variables play in disposing one to alcohol use have also failed to shed significant light on the matter (Russell and Mehrabian, 1975; Kilpatrick, Sutker and Smith, 1976).

Several human experimental models have been employed to test the TRT. In one model, individuals are exposed to various levels of stress, and given ad libitum access to alcohol. Amount or rate of ethanol consumption is the outcome measure (e.g. Allman, Taylor and Nathan, 1972; Higgins and Marlatt, 1975; Marlatt et al., 1975; Holroyd, 1978). The TRT is supported if higher levels of ethanol intake follow more intense stress. In a second model, individuals are overtly or surreptitiously given various quantities of ethanol, and the $S \mathrm{~s}$ reactions to a standardized stress-induction procedure are measured (e.g. Wilson and Abrams, 1977; Abrams and Wilson, 1979; Keane, Lisman and Kreutzer, 1980; Sutker, Allain, Brantley and Randall, 1982). In this approach. ethanol intoxication is the independent variable and intensity of stress response is the

\footnotetext{
*Portions of this paper were presented at the A. Meet. of the Association for Behavior Analysis, Milwaukee. Wisc., 26-29 May 1982.

† $\mathrm{T}$ whom all correspondence should be addressed at: School of Social Work, Florida State University, Tallahassee,

FL 32306. U.S.A. 
outcome measure. If decreased stress is associated with increased levels of intoxication, the TRT is supported.

Though the logic of these approaches is sound, methodological problems and confounding factors preclude clear-cut conclusions about the antianxiety effects of alcohol. For example. a $S$ 's rate of ethanol consumption can influence the drug's physiological, motoric, cognitive and affective effects (Jones and Vega, 1973; Moskowitz and Burns, 1976; Connors and Maisto. 1979). A $S$ 's expectations of alcohol's effects and his drinking history may also alter his responses to ethanol (Berg, Laberg, Skutle and Ohman, 1981; Abrams and Wilson, 1979; Marlatt. 1979). Wilson's (1982) review of this literature highlights two other difficulties. Most studies in which a $S$ 's stress response is the dependent variable have not employed comprehensive behavioral assessment methodology. Given the known desynchrony which often occurs in an individual's motoric, subjective and physiological responses to stress (Rachman and Hodgson, 1974; Hodgson and Rachman. 1974: Grey, Sartory and Rachman, 1979), studies that measure only one or two of these responses may inadequately assess the effects of ethanol. This criticism applies to most cxperiments on the TRT. A second major problem noted by Wilson (1982) is the uncertainty of the stress-induction procedures. Most studies do not ensure that the stressor induces the same amount of anxiety in various $S \mathrm{~s}$, and in the absence of such a demonstrably reliable stress-induction procedure, a study may be questioned as a true test of the TRT.

The present investigation employed a credible stress-induction procedure and systematic assessment of multiple aspects of the patient's anxiety response. The stressor was administering a behavioral approach test (BAT) to clinically phobic patients, a well-documented technique for inducing severe but short-lived anxiety (Levis. 1969). It consists of encouraging a phobic person to allow his/her specific anxiety-evoking stimulus (snake, rat, insect etc.) to be brought closer and closer, along a preplanned series of distances. Simultaneously, subjective and physiological indices of anxiety can be measured. The promise of this methodology for advancing research into anxiety has been previously noted:
"Psychological, physiological and pharmacological studies of acute human anxiety have been seriously limited in the past due to lack of methods for reliably producing acute anxiety under controlled conditions. If the results did not come out as predicted, doubt would be cast upon them because of the possibility that the anxiety was not powerful enough or not 'real' enough. If studies of flooding are carefully planned and designed, these limitations are all overcome. There can be little doubt that the anxiety is real. It is usually powerful and can be turned off at will... Flooding would appear to be an excellent test system for antianxiety drugs or for drug modification of specific components of anxiety." (Curtis, Nesse, Buxton, Wright and Lippman, 1976, p. 159)

See also the similar comments by Blackwell and Whitehead (1975).

Despite these suggestions, BATs have rarely been used in humans to evaluate the antianxiety effects of pharmacological agents. Whitehead, Blackwell and Robinson (1978) employed BATs with 14 analog phobic $S$ s in a double-blind, placebo-controlled study of the antianxiety effects of diazepam. Behavioral approach was closer and subjective anxiety lower for the diazepam $S$ s. These results supported the anxiolytic effects of diazepam and of their use for the reduction of situational fears.

Only one study has employed BATs to test the TRT of alcohol abuse (Rimm, Briddell, Zimmerman and Caddy, 1981) with the additional use of a balanced placebo design to partially control for the effects of alcohol-related expectancies (Marlatt, 1979). The $S$ s were 56 undergraduate student analog phobic volunteers reporting snake fears. Following the ingestion of the experimental beverage, the low-demand BAT was conducted for $S \mathrm{~s}$ in each of the four groups of the balanced placebo design. $S$ s actually receiving alcohol reached a blood alcohol level (BAL) criterion of $0.04 \%$. Ethanol intoxication decreased subjective anxiety but had no effect on observed behavioral approach during the BATs. The expectancy manipulation had no discernible effect. These desynchronous results failed to shed much light on the validity of the TRT. The present study was conducted to provide a more critical test of the antianxiety effects of ethanol by using a higher BAL in clinically phobic patients who were subjected to a high-demand BAT. 


\section{METHOD}

\section{Subjects}

Twenty-two $S$ s were recruited from patients seeking treatment for specific phobias at the Anxiety Disorders Program of the Department of Psychiatry at the University of Michigan Hospitals. Ss were offered limited free treatment in exchange for participation in the study. Each patient completed the standard Anxiety Disorders Program packet of assessment questionnaires. This packet includes an extensive phobia-assessment questionnaire, family history forms, a medical history questionnaire, the Michigan Alcoholism Screening Test (MAST; Seltzer, 1971), the Fear Survey Schedule (FSS; Wolpe and Lang, 1969), the State-Trait Anxiety Inventory (STAI; Spielberger, Gorsuch and Lushene, 1970), the Symptom Checklist-90 (SCL-90; Derogatis, Lipman and Covi, 1973), standardized rating scales for phobic avoidance and of impairment in life functioning due to the phobia (Marks and Matthews, 1979) and inventories assessing agoraphobic symptomatology and panic disorder. Patients then had a 1 -hr diagnostic interview with the senior author. If the presence of a DSM-III defined diagnosis of Simple Phobia was confirmed, a determination was made of the $S$ 's suitability for participation in the experiment. The final sample consisted of 2 men and 20 women, who ranged in age from 20 to $54 \mathrm{yr}$, with a mean of $30 \mathrm{yr}$. Age of onset for their fear ranged from 3 to $19 \mathrm{yr}$, with a mean of $7 \mathrm{yr}$. Nineteen of the $S \mathrm{~s}$ were Caucasian, 2 were Black and 1 was Oriental. Six had phobias for spiders, 6 for snakes, 3 for birds, 2 for crickets, 2 for worms, 2 for rats and 1 for birds. The mean score on the Marks and Matthews (1979) Scale for Behavioral Avoidance was 7 (out of a maximum of 8), corresponding to a self-report rating between 'markedly avoid' and 'always avoid'. Mean impairment in daily living was rated as 5 (out of a maximum of 8), which falls between 'definitely disturbing/disabling' and 'markedly disturbing/disabling'. Psychometric data from the STAI, FSS, the SCL-90 and its subscales and the MAST, indicated a general absence of severe psychopathology, a finding confirmed by clinical interview.

Subjects suitable for participation were given a complete description of the experiment and an opportunity to ask questions before giving informed consent.

\section{Experimental design}

After an initial baseline phase of $15 \mathrm{~min}$, patients took a standardized BAT under high-demand conditions which encouraged maximum possible approach in order to induce a maximum level of anxiety (Evans, 1975; Hastings and Walker, 1975). Patients then consumed either the placebo beverage or the intoxicating beverage, and then waited $20 \mathrm{~min}$ during a second baseline period. After breathalyzer testing to confirm the level of intoxication, the second BAT was carried out, followed by a final 20 -min baseline. A schematic of the experimental design is presented in Fig. 1.

\section{Dependent measures}

Self-report. The $S$ 's level of anxiety was repeatedly assessed using Wolpe's (1973) Subjective Units of Distress Scale (SUDS). The $S$ was asked to rate his or her present level of fear on a 0-100 point scale, with 0 representing complete relaxation and 100 a state of sheer terror or panic. The SUDS procedure is commonly employed in anxiety research and it is known to correlate highly with several indices of autonomic arousal (Thyer, Papsdorf, Davis and Vallecorsa, 1984). A version of Borkovec's (1976) Autonomic Perception Questionnaire (APQ) was used to assess the S's perceived gastrointestinal, cardiovascular, respiratory and muscular arousal before and after each BAT and at the end of the experiment.

\section{Behavioral measures}

Phobic anxiety evoked by real-life exposure to the feared stimulus was measured with a standardized BAT as described by Levis (1969). The $S$ was reminded that the animal that (s)he was afraid of was in the next room. Pulse and SUDS were recorded, then the experimenter asked if he could bring the animal into the room. Latency to agree with this request was recorded, then the experimenter left the room and returned with the phobic animal (evoking stimulus, ES) appropriately restrained (caged. leashed etc.) at a distance of $15 \mathrm{ft}$. Permission was sought to bring 


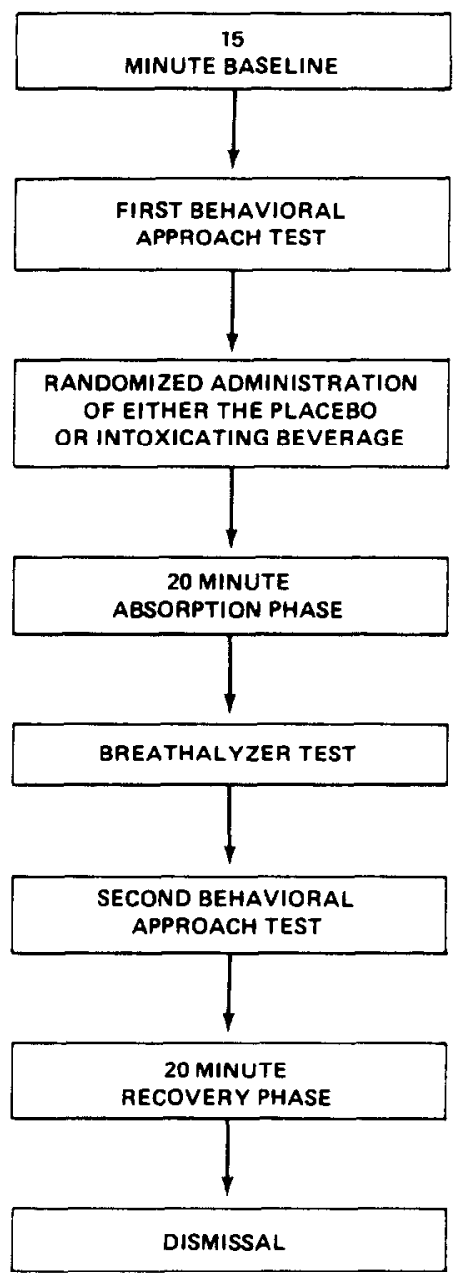

Fig. 1. Experimental design.

the animal closer and closer to the $S$, following standardized increments of proximity. At each step, the $S$ 's pulse, SUDS and latency to comply with the request for closer approach were recorded. When the $S$ refused to allow the animal any closer, final measures were recorded. including an overall experimenter rating of behavioral approach. The rating scalc for behavioral approach is found in Table 1.

The two behavioral measures are therefore: (1) maximum allowable approach of the patient to the ES; and (2) the time (latency) it took for the $S$ to agree with each new requested level of approach.

\section{Physiological measures}

Heart rate (HR) was recorded as a measure of autonomic arousal since previous research has clearly established this measure as reactive to phobic anxiety (Marks, Marset. Boulougouris and Huson, 1971). Pulse was measured with a Gulf and Western HR monitor (Series 4000A), with the photoplethysmograph attached to the right index finger. Blood pressure (BP) was measured using standard sphygmomanometric technique before and after each BAT and at the end of the final baseline period as a second index of cardiovascular reactivity.

\section{Beverage manipulation}

The independent variable consisted of the presence or absence of an intoxicating dose of ethanol in the drink the patient consumed. After the first (sober) BAT, the experimenter left the room and 
Table 1. Behavioral approach scoring for small animal phobias

100 Type duration and degree of handling and exposure is limited only by realistic concern for comfort and safety. Will allow harmless and docile animal complete freedom to move about his/her body. Handles and controls animal confidently and effectively without assistance. Will do all these things for as long or as often as requested. May still show appropriate caution toward animal of uncertain capabilities of temperament.

99 Handles and controls animal, but awkwardly and incompletely, requiring occasional assistance. Allows animal almost unlimited contact between self and animal, but only briefly in some body areas (face etc.)

97 Will tolerate sustained touch at multiple contact points, but excluding several areas (face, inside clothing etc.). Perhaps brief handling and holding with assistance. Animal allowed limited freedom to move about $S$ 's body.

95 Will touch animal but only hesitantly and briefly

90 Will keep unlimited contact with anything and everything which is in contact with the phobic stimulus, even risking the possibility that it might touch him/her by a sudden movement, i.e. inside of container, table.

80 Tolerates extensive contact with things (table, container. stick. leash) which are in contact with the phobic object, but need not risk being contacted if the phobic object should move.

70 Will touch at least briefly something (container, leash. stick) which is in indirect contact with the phobic object.

50 Will approach to within $1 \mathrm{ft}$ of transparent container containing object or animal or animal on a leash.

40 Will approach within $2 \mathrm{ft}$ of container or leashed animal.

30 Will approach within $4 \mathrm{ft}$ of container or leashed animal.

20 Will approach within $7 \mathrm{ft}$ of container.

10 Enters room and remains $10 \mathrm{ft}$ away from object

5 Enters room but withdraws promptly

0 Will not enter room when animal or object is present in a secure container, or on a leash.

the attending psychiatrist gave the $S$ his/her drink accompanied by the following oral instructions:

"This experiment is designed to examine the effects of various amounts of alcohol on differing aspects of anxiety. The beverage you are being asked to consume does contain a certain amount of alcohol. The design of the study does not permit me to tell you the precise amount of alcohol which is in your drink, but needless to say, it is an amount which will not harm you. Please drink this as quickly as possible and try not to reveal to the experimenter how much alcohol you think you consumed."

Subjects assigned on the basis of a coin toss by the psychiatrist to the ethanol intoxication condition were given $0.8 \mathrm{cc}$ of 100 proof vodka/lb of body wt, mixed with orange juice and crushed ice to equal $300 \mathrm{cc}$ of beverage, divided into two cups. Previous research in our laboratory has determined that this dose reliably elevates a fasting $S$ 's blood alcohol level to $0.08 \%$ within 30 min. $S$ s assigned to the placebo condition received $284 \mathrm{cc}$ of crushed ice and orange juice; $7 \mathrm{cc}$ of 100 proof vodka were carefully layered on top of the orange juice to impart the initial sensation of drinking a potent alcoholic beverage. A previous study has shown that $S$ s cannot distinguish this placebo drink from an intoxicating one (Keane et al., 1980). The small amount of vodka ingested by the placebo $S$ s does not produce a detectable elevation in BAL, and the 20 -min period between the completion of beverage consumption and of the breath-testing procedure is of sufficient duration to avoid contamination of the breath test by mouth alcohol (Caddy, Sobell and Sobell, 1978). Beverages were prepared by the psychiatrist in an adjacent room, out of sight from both $S$ and experimenter. Random assignment to the intoxicating or placebo condition was made after the $S$ had arrived. and the assigned condition was not revealed to either the experimenter or the $S$ until the experimental session was concluded.

Use of a complete balanced placebo design was avoided for three reasons. The balanced placebo design would require two additional cells with a doubling of the number of $S$ s required to complete this study. There were not a sufficient number of clinical phobics who met the criteria for this study to permit this. Secondly, several recent studies employing the balanced placebo design found no significant effect for expectancy of the effects of ethanol intoxication (Rimm Sininger, Faherty, Whitley and Perl, 1982; Vuchinich and Tucker, 1980). Lastly, using the BAL employed in this study (0.08 or higher). several studies have found the balanced placebo design difficult to implement (McCarty. 1981: Rohsenow and Marlatt, 1981). Ss who received a dose of ethanol sufficient to elevate their BAL to 0.08 or higher could readily discriminate the internal effects of the drug and 
correctly attributed these effects to the supposedly innocuous beverage they had consumed, thus breaking the expectancy manipulation which is crucial to the balanced placebo design.

\section{Procedure}

After arriving in the laboratory, the patient was weighed by the psychiatrist and then seated in a comfortable chair. The experimenter reviewed study procedures with the patient, answered questions and attached the photoplethysmograph. The experimenter then explained the use of the SUDS scale and ascertained that the scale anchors were understood correctly. After an initial reading including SUDS, HR and BP, SUDS ratings and $H R$ were recorded at 2 -min intervals, during the 15 min first baseline period, after which the patient completed the APQ and BP readings were again recorded.

The experimenter conducted the first BAT using the specific phobic animal which the patient feared and after its conclusion immediately recorded the patient's BP, and administered the second APQ. The patient next consumed the experimental beverage, administered by the psychiatrist after the experimenter left the room. The total drinking time was recorded. After the beverage was consumed, the experimenter returned and began the second baseline phase of $20 \mathrm{~min}$, conducted in a manner similar to the first one. At the end of this 20-min absorption period, the psychiatrist administered a breathalyzer test. A reading of 0.08 or higher was the criterion for proceeding. If a patient who received the intoxicating dose of ethanol was below this criterion, a second breath test was repeated every few minutes until the 0.08 level was reached. Repeated breathalyzer tests were randomly readministered to patients receiving the placebo drink to preserve the double-blind design. The breathalyzer (Intoximeters Inc., Alco-Sensor II) was calibrated before each $S$ 's run and is accurate to within $10 \%$ of actual blood alcohol level.

After the breath testing, the psychiatrist left the room and the experimenter administered the third APQ and recorded the S's BP. The experimenter then conducted the second BAT, employing the same phobic animal as in the first BAT and the same degree of social pressure. At the end of this BAT, the fourth APQ was administered and BP retaken. The third baseline, of 20 min duration, was conducted in a manner similar to the second, concluding with the fifth APQ and a BP reading. At this point, the random assignment code was broken with respect to the experimenter. Any questions the $S$ may have had were answered and participants who received the placebo dose and felt sober were allowed to leave. $S$ s who received the intoxicating dose were given instructions not to drive for $6 \mathrm{hr}$ and were released to a responsible party. Those without someone to escort them waited in a reception room until their BAL was below 0.05 .

\section{RESULTS}

\section{Random assignment manipulation}

There were $10 S \mathrm{~s}$ randomly assigned to the intoxication group and 12 to the placebo group. $\chi^{2}$ Analyses revealed that the two groups did not differ significantly on the categorical variables of gender, race or type of anxiety-evoking stimulus. Similarly, an examination of psychometric characteristics using student $t$-tests also failed to demonstrate any significant differences between $S$ s assigned to the two groups. These data suggest that the random assignment procedure was effective in developing equivalent experimental and placebo groups.

\section{Beverage manipulation}

The $10 \mathrm{~S}$ s assigned the intoxicating beverage received an average dose of $106 \mathrm{cc}$ of 100 proof vodka, while each of the placebo $S$ s consumed $14 \mathrm{cc}$ of vodka. The mean blood alcohol level obtained by the intoxicated $S \mathrm{~s}$ was $0.09 \mathrm{ml} / 100 \mathrm{ml}(\mathrm{SD}=0.03$ ), while no detectable BAL elevations were observed among the placebo $S$ s. Immediately following beverage consumption, each $S$ gave an estimate of the number of fluid ounces of vodka he or she had ingested. The experimental group gave a mean rating of $2.77 \mathrm{fl} . \mathrm{oz}(\mathrm{SD}=1.31$ ), which is equivalent to $82 \mathrm{cc}$, while the $S \mathrm{~s}$ in the placebo condition gave a mean estimate of $1.18 \mathrm{fl} . \mathrm{oz}(\mathrm{SD}=0.41)$, equal to $34 \mathrm{cc}$. This difference, evaluated using a student $t$-test, is significant at less than the 0.001 level. No $S$ gave an estimated rating of 0 n.oz of vodka, indicating the credibility with which they accepted the instructions given them by the psychiatrist. It is not surprising, considering the BAL criterion, that the experimental $S$ s should 


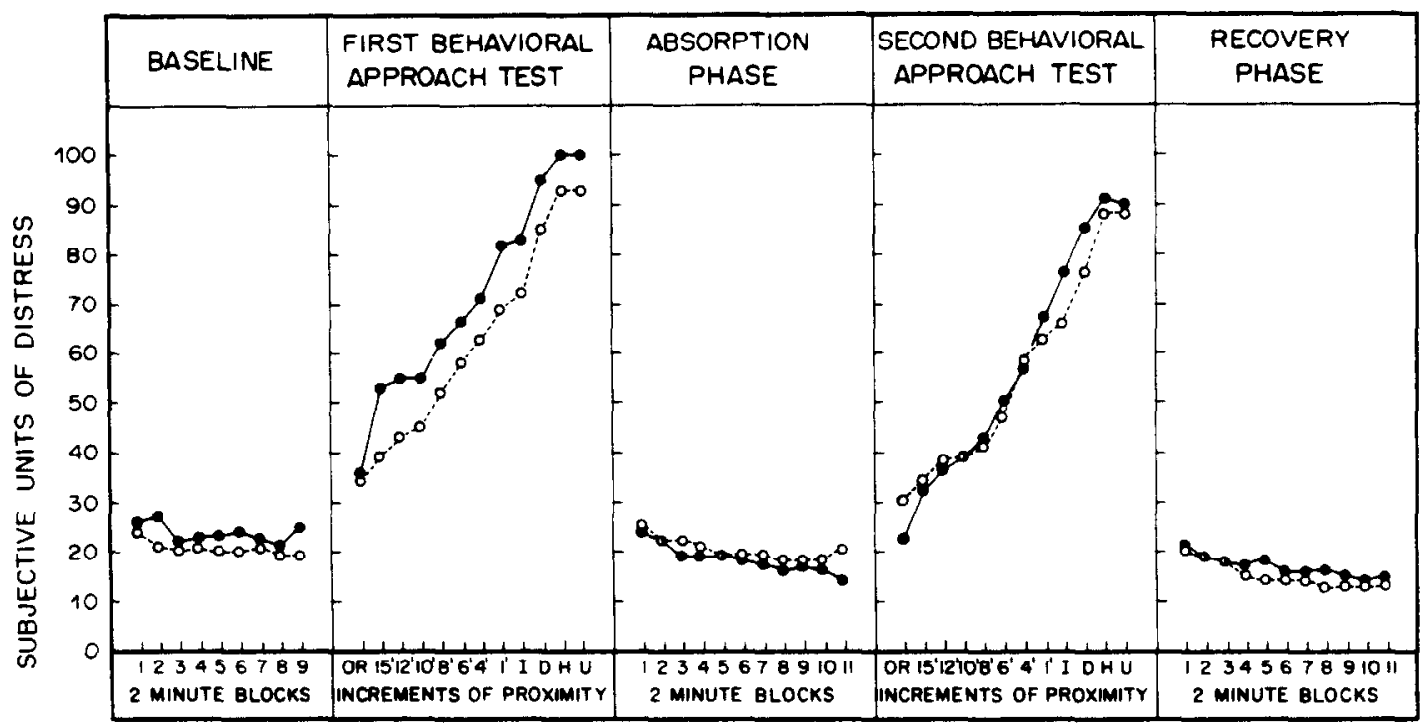

Fig. 2. Mean adjusted SUDS for each sampling period. Abbreviations for the increments of proximity are: $\mathrm{OR}=$ animal out of room; $\mathrm{I}=$ indirect touch; $\mathrm{D}=$ direct touch; $\mathrm{H}=$ hold; $\mathrm{U}=$ unlimited contact. Intoxicated $S \mathrm{~s} ; O$, placebo $S \mathrm{~s}$.

correctly estimate that the dose of vodka they received was significantly higher than dose estimated by the placebo group. What is surprising is that the subjective estimates differed between the two groups by a factor of slightly more than 2 , when in reality the true difference had an order of magnitude of approx. 7. Attained blood alcohol levels among the intoxicated $S$ s ranged from 0.05 to 0.16 . One patient in the intoxication condition reached a BAL of only 0.05 because she took approx. $45 \mathrm{~min}$ to consume her drink, despite the urging of the psychiatrist. She was the only member of the group to be run at a BAL of less than 0.08 .

\section{Self-report measures}

Mean SUDS ratings for each group during the five periods of the experiment (first baseline, first BAT, second baseline, second BAT, third baseline) are displayed in Fig. 2. During the BATs, the more fearful $S$ s were unable to attempt the closer levels of approach. In order to have complete data, the maximum anxiety rating of 100 was substituted for each missing data point representing a level of approach the $S$ refused to attempt. For example, if a $S$ gave a rating of 80 SUDS when $1 \mathrm{ft}$ away from the ES and then refused to allow further contact, a score of 100 was assigned for each of the remaining uncompleted steps of 'indirect touch', 'direct touch', 'hold' and 'unlimited contact".

These adjusted SUDS scores were subjected to a 5 (periods) $\times 2$ (groups) repeated-measures ANOVA. A significant main effect for periods was demonstrated $[F(4,80)=98.67 ; P<0.001]$. No significant main effect was observed for the group factor $[F(1,20)<1 ; P=0.64]$ or for the period $\times$ group interaction $[F(4,80)<1 ; P=0.54]$. The source for the main effect of periods is clearly the BAT procedure. A similar analysis on unadjusted SUDS scores obtained the same results, suggesting that ethanol intoxication did not exert an anxiolytic effect on this aspect of phobic anxiety.

The $S \mathrm{~s}^{\prime}$ degree of perceived autonomic arousal was assessed via their total scores on the APQ, given immediately before and after each BAT and at the end of the last baseline. The APQ data is presented in Table 2.

A 5 (APQ administrations) $\times 2$ (groups) repeated-measures ANOVA on these data revealed a significant main effect for the time of administration $[F(4,72)=4.07 ; P<0.01]$. No significant effects were observed for the group factor $[F(1,18)<1 ; P=0.43]$ or for the period $\times$ group interaction $[F(4.72)=1.21 ; P=0.31]$. Inspection of the data in Table 2 indicates that the reported peaks in autonomic arousal occurred after each BAT. The APQ has a range of scores from 0 to 144 and the observed effects, although statistically significant, appear minor. The BAT appears to 
Table 2. APQ scores

\begin{tabular}{lcrrrr}
\hline & \multicolumn{2}{c}{$\begin{array}{c}\text { Ethanol } S \mathrm{~s} \\
(N=10)\end{array}$} & \multicolumn{2}{c}{$\begin{array}{c}\text { Placebo } S \mathrm{~s} \\
(N=12)\end{array}$} \\
\cline { 2 - 4 } \multicolumn{1}{c}{ Administration } & \multicolumn{1}{c}{$\bar{X}$} & $\mathrm{SD}$ & \multicolumn{1}{c}{$\bar{X}$} & $\mathrm{SD}$ \\
\hline 1. Before first BAT & 13.9 & 8.63 & 12.25 & 7.43 \\
2. After first BAT & 15.8 & 10.82 & 17.41 & 14.78 \\
3. Before second BAT & 10.20 & 8.10 & 7.66 & 6.90 \\
4. After second BAT & 15.00 & 9.43 & 10.58 & 8.73 \\
5. End of experiment & 12.62 & 11.05 & 5.16 & 4.08 \\
\hline
\end{tabular}

somewhat enhance perceived autonomic arousal, but ethanol intoxication does not modify the experience.

\section{Behavioral measures}

Each $S$ 's level of approach during each of the two BATs was rated by the experimenter employing an analog scale ranging from 0 (complete avoidance) to 100 (allowing unlimited contact with the ES within the dictates of safety) (see Table 1). These ratings of behavioral approach were subjected to a 2 (first vs second BAT) $\times 2$ (groups) repeated-measures ANOVA. The analysis revealed a significant main effect for testing: $S$ s demonstrated increased approach on the second BAT $[F(1,20)=7.26 ; P<0.05]$. The group effect was nonsignificant $[F(1,20)<1 ; P=0.92]$, as was the testing $\times$ group interaction $[F(1,20)<1 ; P=0.48]$. These data for behavioral approach are presented in Table 3 and indicate that ethanol intoxication did not appear to reduce behavioral avoidance to a phobic anxiety-evoking stimulus.

For each increment on the BAT, the experimenter recorded the $S$ 's latency to comply with his request for permission to bring the ES a further step closer. An immediate assent was rated as 0 sec and the maximum delay could be $30 \mathrm{sec}$, after which the BAT was terminated. The latency scores were subjected to a 2 (first vs second BAT) $\times 2$ (groups) repeated-measures ANOVA. No significant main effects were observed, nor was the time $\times$ group interaction significant. These results are displayed in Table 4. Ethanol intoxication does not appear to affect the $S$ 's hesitancy to decrease their proximity to a phobic object.

\section{Physiological measures}

Mean HRs for each group during each of the five experimental periods are displayed in Fig. 3. The elevation in HR observed during the two BATs relative to the baselines suggests that the exposure procedure was an effective stressor. HR scores were subjected to a 5 (periods) $\times 2$ (groups) repeated-measures ANOVA which reveals a significant main effect for time periods $[F(1,20)=5.78 ; P<0.001]$. No main group effect was observed $[F(1,20)<1: P=0.87]$ or a groups $\times$ periods interaction $[F(4,80)<1 ; P=0.54]$. These results indicate that ethanol intoxication had no consistent effect on resting $H R$ or on the tachycardia induced by exposure to a phobic anxiety-evoking stimulus. The $S$ 's BP was recorded at the beginning of the first baseline, at the beginning and end of each BAT and at the end of the final baseline, for a total of six readings. The systolic readings were subjected to a 6 (time of reading) $\times 2$ (groups) repeated-measures ANOVA. The readings were not found to significantly change over time. The group effect was nonsignificant as was the time $\times$ groups interaction. The data for diastolic BP was also examined with a repeated-measures ANOVA and a significant main effect was observed for time of recording $[F(5,85)=6.25 ; P<0.001]$. The group factor was nonsignificant while the time $\times$ group interaction just barely excluded significance. The data for BP readings are presented in Table 5 and indicate that the diastolic BP peaked at the beginning of the second baseline, immediately after

\begin{tabular}{ccccc} 
Table 3. Maximum level of approach scores during each BAT $^{-4}$ \\
\hline & \multicolumn{2}{c}{ Ethanol $S_{s}$} & \multicolumn{2}{c}{ Placebo $S \mathrm{~s}$} \\
Administration & $\bar{X}$ & SD & $\bar{Y}$ & SD \\
First BAT & 54.50 & 31.66 & 69.60 & 31.90 \\
Second BAT & 58.83 & 31.00 & 67.58 & 30.03 \\
\hline
\end{tabular}

${ }^{4}$ See Table I for scoring criteria 
Table 4. Mean latency to comply with each step of the BAT (sec)

\begin{tabular}{|c|c|c|c|c|c|c|}
\hline \multirow[b]{2}{*}{ Level of approach } & \multicolumn{3}{|c|}{ Ethanol $S \mathrm{~s}$} & \multicolumn{3}{|c|}{ Placebo $S \mathbf{s}$} \\
\hline & $N$ & $\bar{x}$ & SD & $N$ & $\bar{X}$ & SD \\
\hline & \multicolumn{6}{|c|}{ First $B A T$} \\
\hline $15^{\prime}$ & 10 & 0.00 & 0.00 & 12 & 0.00 & 0.00 \\
\hline $12^{\prime}$ & 10 & 2.20 & 6.26 & 12 & 0.91 & 1.56 \\
\hline $10^{\prime}$ & 10 & 2.40 & 6.25 & 12 & 2.83 & 4.87 \\
\hline 8 & 10 & 1.90 & 3.28 & 12 & 3.50 & 5.28 \\
\hline $6^{\prime}$ & 8 & 4.87 & 8.82 & 11 & 3.45 & 7.06 \\
\hline $4^{\prime}$ & 7 & 1.00 & 1.00 & 8 & 1.50 & 3.50 \\
\hline $1^{\prime}$ & 6 & 5.83 & 7.80 & 7 & 1.42 & 1.61 \\
\hline Indirect touch & 4 & 6.25 & 2.50 & 6 & 2.00 & 4.00 \\
\hline Direct touch & 2 & 20.00 & 7.07 & 3 & 7.66 & 4.04 \\
\hline Hold & 0 & - & - & 2 & 15.00 & 21.21 \\
\hline \multirow[t]{2}{*}{ Unlimited contact } & 0 & - & - & 2 & 12.50 & 17.67 \\
\hline & \multicolumn{6}{|c|}{ Second $B A T$} \\
\hline $15^{\prime}$ & 10 & 0.00 & 0.00 & 12 & 0.16 & 0.57 \\
\hline $12^{\prime}$ & 10 & 0.00 & 0.00 & 12 & 0.16 & 0.57 \\
\hline $10^{\prime}$ & 10 & 0.00 & 0.00 & 12 & 0.25 & 0.86 \\
\hline $8^{\prime}$ & 10 & 1.00 & 2.10 & 12 & 0.58 & 1.50 \\
\hline $6^{\prime}$ & 10 & 2.00 & 4.83 & 12 & 2.50 & 5.00 \\
\hline $4^{\prime}$ & 9 & 3.55 & 6.52 & 10 & 4.20 & 8.01 \\
\hline $1^{\prime}$ & 7 & 2.42 & 2.50 & 9 & 5.11 & 6.52 \\
\hline Indirect touch & 6 & 1.16 & 2.04 & 7 & 1.42 & 2.43 \\
\hline Direct touch & 5 & 13.00 & 5.70 & 5 & 5.40 & 6.76 \\
\hline Hold & 3 & 15.00 & 8.66 & 2 & 1.00 & 1.41 \\
\hline Unlimited contact & 3 & 10.00 & 0.00 & 2 & 1.00 & 1.41 \\
\hline
\end{tabular}

the first BAT. The marginal interaction effect was not unexpected since it is known that ethanol influences cardiovascular activity (Knott and Beard, 1971). The changes were in the direction of increased diastolic BP in the intoxicated $S \mathrm{~s}$, which is not congruent with the predicted anxiolytic effects.

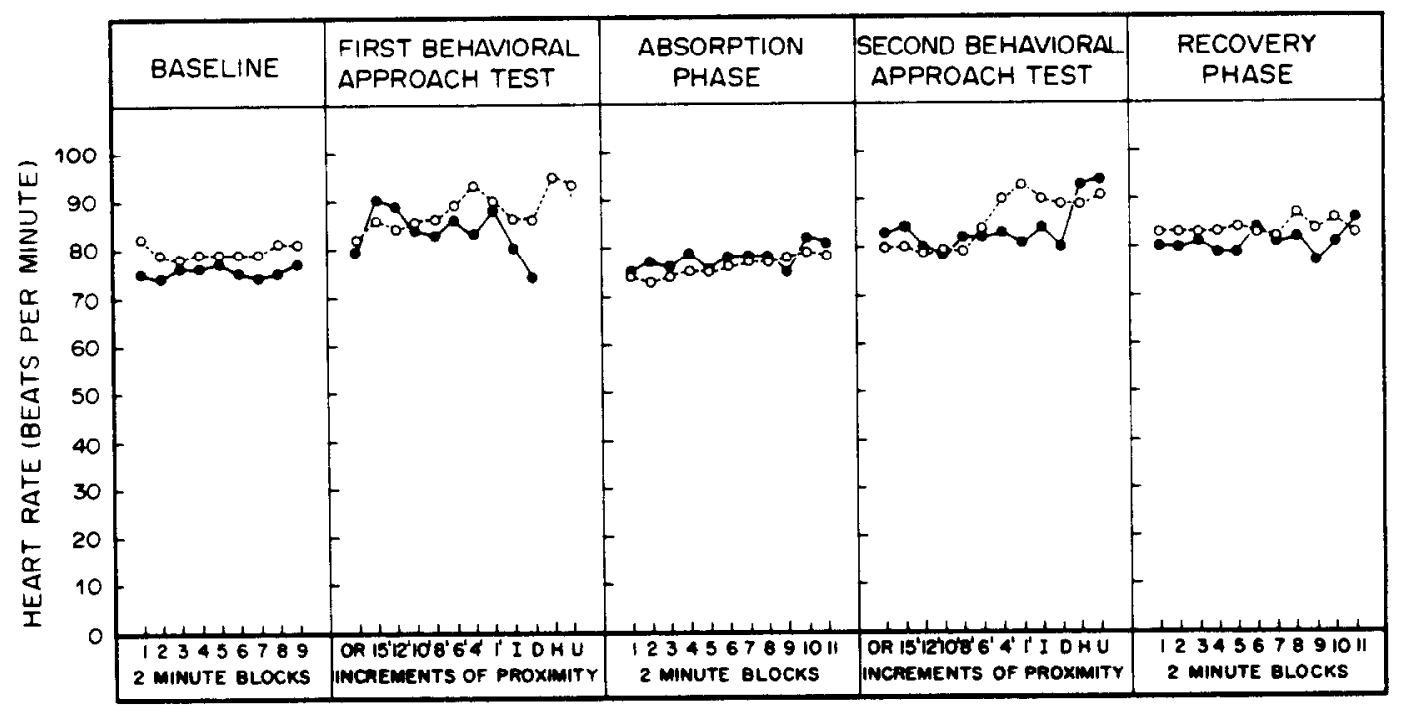

Fig. 3. Mean HR for each sampling period. Abbreviations as given in Fig. 2.

Table 5. BP values $(\mathrm{mmHg})$

\begin{tabular}{|c|c|c|c|c|c|c|c|c|}
\hline \multirow[b]{3}{*}{ Time } & \multicolumn{4}{|c|}{ Ethanol $S$ s } & \multicolumn{4}{|c|}{ Placebo $S$ s } \\
\hline & \multicolumn{2}{|c|}{ Systolic } & \multicolumn{2}{|c|}{ Diastolic } & \multicolumn{2}{|c|}{ Systolic } & \multicolumn{2}{|c|}{ Diastolic } \\
\hline & $\bar{X}$ & $\overline{\mathrm{SD}}$ & $\bar{X}$ & SD & $\bar{X}$ & $\mathrm{SD}$ & $\bar{X}$ & SD \\
\hline Beginning of experiment & 118 & 13 & 73 & 7 & 103 & 33 & 75 & 5 \\
\hline Before first BAT & 109 & 13 & 73 & 8 & 109 & 20 & 74 & 5 \\
\hline After first BAT & 114 & 12 & 80 & 7 & 109 & 11 & 78 & 8 \\
\hline Before second BAT & 112 & 14 & 73 & 5 & 111 & 10 & 75 & 8 \\
\hline After second $\mathrm{BAT}$ & 111 & 12 & 76 & 7 & 107 & 11 & 74 & 7 \\
\hline End of experiment & 110 & 8 & 77 & 5 & 102 & 8 & 72 & 7 \\
\hline
\end{tabular}




\section{Summary of Results}

In the present context of a carefully designed double-blind study of the anxiolytic effects of alcohol, employing an effective stress manipulation, ethanol intoxication was not found to exert any antianxiety effects on subjective fear, behavioral avoidance. HR. BP or perceived autonomic arousal. This synchronous pattern of results suggests that the fundamental tenet of the TRT of alcohol abuse may not be valid.

\section{DISCUSSION}

The results of this experiment seem clear-cut ethanol intoxication does not reduce phobic anxiety. This contradicts a fundamental assumption of the TRT. Phobic anxiety, although somewhat diminished in both groups during the second BAT, was not decreased to a greater extent in the intoxicated $S$ s relative to the placebo group. Accordingly, the present study contributes a further piece of evidence toward the falsification of the TRT. The robustness of this conclusion seems rather strong. The construct of anxiety was assessed through concurrent multiple measures employing all three response channels. In each instance, ethanol intoxication failed to exhibit the predicted antianxiety effect. The synchrony or consensus of these results supports this conclusion.

It could be argued that a large dose of ethanol, producing a higher blood alcohol level, would reduce phobic anxiety. The present study employed a BAL criterion of $0.08 \mathrm{mil}$ of ethanol per $100 \mathrm{ml}$ of blood, a dose sufficient to render the $S$ legally intoxicated. A study using a lower BAL has reported a partial anxiolytic effect; hence there is reason to suspect that the 0.08 criterion seems not to be too low (Rimm et al., 1981). Also, a still higher dose would have increased the attendant complications of nausea, agitation, loss of coordination and simple sedation, as distinguished from any anxiolytic effect. When compared to earlier research, the $0.08 \mathrm{BAL}$ represents one of the higher levels of intoxication which have been employed in experimental inquiries on the TRT.

Ethanol intoxication might be argued to have an anxiolytic 'therapeutic window' or BAL range at which tension reduction is apparent, although higher or lower doses fail to exert such an effect. This may be valid. Rinm et al. (1981), employing a similar methodology but with a BAL of 0.04 (half that of the present study), found intoxication to reduce the self-report of fear, but not to reduce phobic avoidance. However, the TRT does not postulate such a therapeutic window, and if such an effect were actually to be in operation, the careful degree of self-titration required by an alcohol consumer renders this alternative rather implausible.

A methodologist might suggest that the balanced placebo design would have been desirable to further isolate the relative contribution of alcohol and expectancies to any observed effects. Prior studies using such a design with a BAL similar to that employed in the present experiment were unable to maintain a credible beverage manipulation. $S$ s adequately discriminated their internal cues and correctly guessed whether or not they received ethanol. thus destroying the expectancy manipulation (Bradlyn, Strickler and Maxwell, 1981; Connors and Maisto, 1979). The present study omitted two cells of the balanced placebo design, a group who neither expected nor received ethanol, and a group who expected nothing but were covertly administered an intoxicating dose of ethanol. Assigning $S$ s to the former condition would be useful in an experiment on the BAT procedure and how it is modified by repetition but has little bearing on the question of how ethanol modifies anxiety responses. Assigning $S$ s to the latter condition would likely prove a waste of time since at a BAL of 0.08 they could tell that they had received some sort of pharmacological agent. Such an experimental condition would also require deception, in terms of rendering psychiatric patients intoxicated when they had not given informed consent to such procedure.

Each of the dependent measures in the present study are well documented in terms of their relationship to the construct of anxiety. Additional measures could have been employed such as electrodermal responses or scores on a state anxiety inventory given pre and post each BAT. The measures actually selected represented a compromise between adequate vs overly complex assessment procedures. Only a few previous investigations on the TRT have employed such a comprehensive battery of dependent measures as in the present experiment.

No assessment was made of the $S$ 's expectations or beliefs regarding the antianxiety effects of ethanol. Self-report studies indicate that a large number of individuals believe that ethanol does 
reduce tension (Farber, Khavari and Douglas, 1980). This omission is not likely to have resulted in any significant bias in the composition of the two experimental groups. Following random assignment, the two groups were not found to significantly differ on any other demographic or psychometric characteristics. Individuals holding strong beliefs one way or another with respect to the effects of ethanol were likely to be similarly distributed.

The degree to which the findings of this study can be generalized cannot be determined at present. Several limitations are evident. A very unique form of anxiety was employed as a situational test, phobic anxiety. This may or may not be similar in quality to more vague constructs such as tension, or to other varieties of anxious experience such as those employed in previous research on the tension reduction theory. The demand for experimental rigor and highly operational dependent variables, while increasing internal validity, simultaneously exerted a restricting influence on the external validity of the study. It should be conservatively noted accordingly that ethanol intoxication was not found to reduce phobic anxiety. Extrapolation to other states of anxiousness may not be justified. Indeed, these results may only be valid for Simple Phobia, and not for Social Phobia or Agoraphobia.

The TRT is an explanation of alcohol abuse yet nonaddicted $S$ s were employed. Generalizing the results obtained in the present study to alcoholics may be unwarranted. As Cappell and Herman (1972) pointed out, the TRT actually consists of two separate hypotheses: (1) that ethanol possesses antianxiety effects; and (2) that individuals learn to drink alcohol to reduce anxiety. The present study actually tested on the first of these two hypotheses, and failed to support it, adding to a growing body of research which suggests that the fundamental premise of the TRT of alcoholism may not be valid. This lack of consensus suggests that treatment regimens based upon the assumption that alcohol abusers drink in order to reduce tension may be inappropriate for a large number of patients.

Acknowledgements - This project was supported in part by research grants from the Distilled Spirits Council of the United States and from the Michigan Chapter of the National Council on Alcoholism.

The authors would like to acknowledge the editorial assistance of Randolph Nesse and Oliver Cameron in preparing this report.

\section{REFERENCES}

Abrams D. and Wilson G. (1979) Effects of alcohol on social anxiety in women: cognitive versus physiological processes. J. abnorm. Psychol. 88, 161-173.

Allman L., Taylor H. and Nathan P. (1972) Group drinking during stress: effects on drinking behavior, affect and psychopathology. Am. J. Psychiat. 139, 669-678.

Berg G., Laberg J.. Skutle A. and Ohman A. (1981) Instructed versus pharmacological effects of alcohol in alcoholics and social drinkers. Behat. Res. Ther. 19, 55-66.

Blackwell B. and Whitehead W. (1975) Behavioral evaluation of antianxiety drugs. In Predictability in Psychopharmacology: Preclinical and Clinical Correlations (Edited by Sudilovsky A., Gershon S. and Beer B.), pp. 121-141. Raven Press, New York.

Borkovec T. (1976) Physiological and cognitive processes in the maintenance and reduction of fear. In Consciousness and Self Regulation (Edited by Schwartz G. and Shapiro D.), pp. 261-308. Plenum Press, New York.

Bradlyn A.. Strickler D. and Maxwell W. (1981) Alcohol, expectancy and stress: methodological concerns with the expectancy design. Addict. Behat. 6, 1-8.

Caddy G. Sobell M. and Sobell L. (1978) Alcohol breath tests: criterion times for avoiding contamination by mouth alcohol. Behat. Res. Meth. Instrumn 10, 814-818.

Conger J. (1956) Alcoholism: theory, problem and challenge- II. Reinforcement theory and the dynamics of alcoholism. Q. Jl Stud. Alcohol 17, 296-305.

Connors G. and Maisto S. (1979) Effects of alcohol, instructions and consumption rate on affect and physiological sensations. Psvchopharmacology 62, 261-266.

Curtis G.. Nesse R.. Buxton M.. Wright J. and Lippman D. (1976) Flooding in vivo as a research tool and treatment method for phobias: a preliminary report. Compreh. Psychiat. 11, 101-107.

Derogatis L.. Lipman R. and Covi L. (1973) SCL-90: an outpatient psychiatric rating scale-preliminary report. Psychopharmac. Bull. 19, 13-28.

Evans M. (1975) Procedures for a high demand behavioral approach test and for a diagnosis/treatment subject expectancy manipulation: a brief note. Behat. Ther. 6, 72-77.

Farber P.. Khavari K and Douglas F. (1980) A factor analytic study of reasons for drinking: empirical validation of positive and negative reinforcement dimensions. J. consult. clin. Psychol. 48, 780-781.

Grey S.. Sartory G. and Rachman S. (1979) Synchronous and desynchronous changes during fear reduction. Behav. Res. Ther. 17, 137-147.

Hastings J. and Walker M. (1975) The effects of level of fear and rate of approach on cardiac rate and avoidance of a phobic stimulus. Behat. Ther. 6, 445-452. 
Higgins R. and Marlatt G. (1975) Fear of interpersonal evaluation as a determinant of alcohol consumption in male social drinkers. J. abnorm. Psychol. 84, 644-651.

Hodgson R. and Rachman S. (1974) Desynchrony in measures of fear. Behar. Res. Ther. 12, 319-326.

Hodgson R., Stockwell T. and Rankin H. (1979) Can alcohol reduce tension? Behat. Res. Ther, 17, 459-466

Holroyd K. (1978) Effects of social anxicty and social evaluation on beer consumption and social interactions. J. Stud. Alcohol 39, 737-744.

Jones B. and Vega A. (1973) Fast and slow drinkers: blood alcohol variables and cognitive performance. Q. Jl Stud. Alcohol 34, 797-806.

Keane T., Lisman S. and Kreutzer J. (1980) Alcoholic beverages and their placebos: an empirical evaluation of expectancies Addict. Behav. 5, 313-328.

Kilpatrick D., Sutker P. and Smith A. (1976) Deviant drug and alcohol use: the role of anxiety, sensation seeking and other personality varıables. In Emotions and Anxiety (Edited by Zuckerman M. and Spielberger C.), pp. 247-278. Wiley, New York.

Kilpatrick D., Sutker P., Best C. and Allain A. (1980) Acute alcohol intoxication and vicarious emotional responsiveness. Addict. Behav. 5, 191-198.

Kingham R. (1958) Alcoholism and the reinforcement theory of learning. Q. Jl Stud. Alcohol 19, 320-330.

Levis D. (1969) The phobic test apparatus: an objective measure of human avoidance behavior to small objects. Behar. Res. Ther. 7, 309-315.

Marks I. and Mathews A. (1979) Brief standard self-ratings for phobic patients. Behav. Res. Ther. 17, $263-267$.

Marks I., Marset P., Boulougouris J. and Huson J. (1971) Physiological accompaniments of neutral and phobic imagery. Psychol. Med. 1, 299-307.

Marlatt G. (1979) Alcohol use and problem drinking: a cognitive behavioral analysis. In Cognitive-Behavioral Intervention: Theory, Research and Procedure (Edited by Kendall P. and Hallon S.). Academic Press. New York.

McCarty D. (1981) Misattribution of ethanol intoxication. Addict. Behav. 6, 369-375.

Moskowitz H. and Burns M. (1976) Effects of rate of drinking on human performance. J. Stud. Alcohol 37, 598-605.

Rachman S. and Hodgson R. (1974) Synchrony and desynchrony in fear and avoidance. Behal. Res. Ther. 12, $311-326$.

Rimm D., Briddell D., Zimmerman M. and Caddy G. (1981) The effects of alcohol and the expectancy of alcohol on snake fear. Addict. Behav. 6, 47-51.

Rimm D., Sininger R., Faherty J., Whitley M. and Perl M. (1982) A balanced placebo investigation of the effects of alcohol versus alcohol expectancy on simulated driving behavior Addict. Behav. 7, 27-32.

Rohsenow D. and Marlatt G. (1981) The balanced placebo design: methodological considerations. Addict. Behat. 6, $107-122$.

Russell J. and Mehrabian A. (1975) The mediating role of emotions in aicohol use. J. Stud. Alcohol 36, 1508-1536.

Seitzer M. (1971) The Michigan Alcoholism Screening Test: the quest for a new diagnostic instrument. Am. J. Psychiat. 127, $1653-1658$.

Shoben E. (1956) Views on the etiology of alcoholism-III. The behavioristic view. In Alcoholism as a Medical Problem (Edited by Druse H.), pp. 47-55. Hoeber-Harper. New York.

Spielberger C., Gorsuch R. and Lushene R. (1970) The State-Trait Anxiety (STAI) Manual for Form X. Consulting Psychologists Press, Palo Alto, Calif.

Sutker P., Allain A., Brantley P. and Randall C. (1982) Acute alcohol intoxication, negative affect and autonomic arousal in women and men. Addict. Behav. 7, 17-25.

Thyer B., Papsdorf J.. Davis R. and Vallecorsa S. (1984) Autonomic correlates of the Subjective Anxiety Scale. J. Behav. Ther. exp. Psychiat. 15, 3-7.

Vuchinich R. and Tucker J. (1980) A critique of cognitive labeling explanations of the emotional and behavioral effects of alcohol. Addict. Behav. 5, 179-188.

Whitehead W. Blackwell B. and Robinson A. (1978) Effects of diazepam on phobic avoidance behavior and phobic anxiety. Biol. Psychiat. 13, 59-64.

Wilson G. T. (1982) Alcohol and anxiety: recent evidence on the tension reduction theory of use and abuse. In Self-control and Self-modification of Emotional Behavior (Edited by Blankstein K. and Polivy J.). Plenum Press, New York.

Wilson G. T. and Abrams D. (1977) Effects of alcohol on social anxiety and physiological arousal: cognitive versus pharmacological processes. Cog. Ther. Res. 1, 195-210.

Wolpe J. (1973) The Practice of Behavior Therapy. Pergamon Press, New York.

Wolpe J. and Lang P. (1969) Fear Survey Schedule. EdITS. San Diego. Calif. 